\title{
Observations of the filamentation of high-intensity laser-produced electron beams
}

\author{
M. S. Wei, ${ }^{1}$ F. N. Beg, ${ }^{1, *}$ E. L. Clark ${ }^{1,2}$ A. E. Dangor, ${ }^{1}$ R. G. Evans, ${ }^{1,2}$ A. Gopal, ${ }^{1}$ K. W. D. Ledingham, ${ }^{3,2}$ \\ P. McKenna, ${ }^{3}$ P. A. Norreys, ${ }^{4}$ M. Tatarakis, ${ }^{1, \dagger}$ M. Zepf, ${ }^{5}$ and K. Krushelnick ${ }^{1}$ \\ ${ }^{1}$ The Blackett Laboratory, Imperial College London SW7 2BZ, United Kingdom \\ ${ }^{2}$ Plasma Physics Department, AWE plc, Aldermaston, Reading RG7 4PR, United Kingdom \\ ${ }^{3}$ Department of Physics, University of Strathclyde, Glasgow G4 ONG, United Kingdom \\ ${ }^{4}$ Central Laser Facility, Rutherford Appleton Laboratory, Chilton, Oxon OX11 OQX, United Kingdom \\ ${ }^{5}$ Department of Physics, The Queen's University, University Road, Belfast BT7 1NN, United Kingdom \\ (Received 16 July 2003; revised manuscript received 30 July 2004; published 23 November 2004)
}

\begin{abstract}
Filamented electron beams have been observed to be emitted from the rear of thin solid targets irradiated by a high-intensity short-pulse laser when there is low-density plasma present at the back of the target. These observations are consistent with a laser-generated beam of relativistic electrons propagating through the target, which is subsequently fragmented by a Weibel-like instability in the low-density plasma at the rear. These measurements are in agreement with particle-in-cell simulations and theory, since the filamentation instability is predicted to be dramatically enhanced when the electron beam density approaches that of the background plasma.
\end{abstract}

DOI: 10.1103/PhysRevE.70.056412

PACS number(s): 52.38.Hb, 52.35.Qz, 52.40.Mj

\section{INTRODUCTION}

The study of laser-generated hot electron beams and their propagation in plasma has received considerable attention recently because of potentially important applications-in particular, the "fast ignition" scheme for inertial fusion energy $[1,2]$. In this concept, a relativistic electron beam produced by a picosecond-duration, high-intensity petawatt laser pulse penetrates into the compressed core of a deuterium/ tritium pellet where it deposits its energy and generates a fusion spark. Compared to conventional inertial confinement fusion, fast ignition is predicted to significantly reduce the energy required for the initial compression and relax the stringent symmetry requirements for irradiation of the pellet. Recent cone-guided fast-ignition experiments are particularly encouraging in this respect [2]. However, since all of the previous experiments have been performed with laser systems much smaller than those which would be necessary for actual ignition experiments, the required laser needs to have energies of tens of $\mathrm{kJ}$ with a pulse duration of about $10 \mathrm{psec}$. There are many issues with regard to the transport of highcurrent multi-Mega-Ampere (multi-MA) laser-produced electron beams in plasma which remain to be investigated.

During intense laser-solid interactions highly directional, high-current electron beams $[3,4]$ can be generated via classical and Brunel-type resonance absorption [5] as well as via ponderomotive $\mathbf{J} \times \mathbf{B}$ acceleration [6]. As such beams penetrate into high-density plasma, a return current is induced in the cold background plasma to compensate for space charge and to allow beams with currents larger than the Alfvén limit

\footnotetext{
*Present address: Department of Mechanical and Aerospace Engineering, University of California, San Diego, San Diego, CA 92093-0411, USA.

†Present address: Institute of Matter Structure and Laser Physics, Technical University of Crete, 73100 Chania, Crete, Greece.
}

$\left(I_{A} \sim 17 \beta \gamma \mathrm{kA}\right)$ to propagate. Such counterpropagating electron streams are subject to the two-stream instability and a Weibel-like filamentation instability [7]. The two-stream instability results in the generation of longitudinal Langmuir turbulence and the filamentation instability produces strong magnetic fields transverse to the fast electron beam, causing the electron beam to break up into small beamlets with a typical transverse dimension of the order of the collisionless skin depth, $c / \omega_{p}$ (where $c$ is the speed of light and $\omega_{p}$ is the electron plasma frequency).

Such instabilities have been the subject of theoretical investigations. The growth rate of the Weibel instability has been studied by linear analysis of the two-fluid equations $[8,9]$. In addition, many computational simulations have been performed and a characteristic result is the breakup of the beam into many current filaments in the plasma, the filaments being guided by quasistatic magnetic fields. These small filaments have been subsequently observed to coalesce [9-11].

In high-intensity laser-solid interaction experiments, collimated electron beams have been observed in the interior of transparent glass targets [12] and at the rear surface of thin solid targets with optical probes [13]. Measurements of proton emission at the rear of the target have also been used to examine indirectly electron beam propagation effects in solid density plasmas $[14,15]$. However, filamentary structures in the electron beams leaving the target plasma have not been reported previously and the experimental regimes in which this instability is important have not been explored.

In this paper, we present the first direct experimental observation of the structure of the hot electron beam emitted in laser-solid target interactions. We have found that large numbers of small-scale filaments (having a dimension approximately that of the plasma skin depth) in the electron beam are always present when the electron beam propagates through a relatively long-scale-length, low-density plasma. This plasma was created at the rear surface of the target by a 


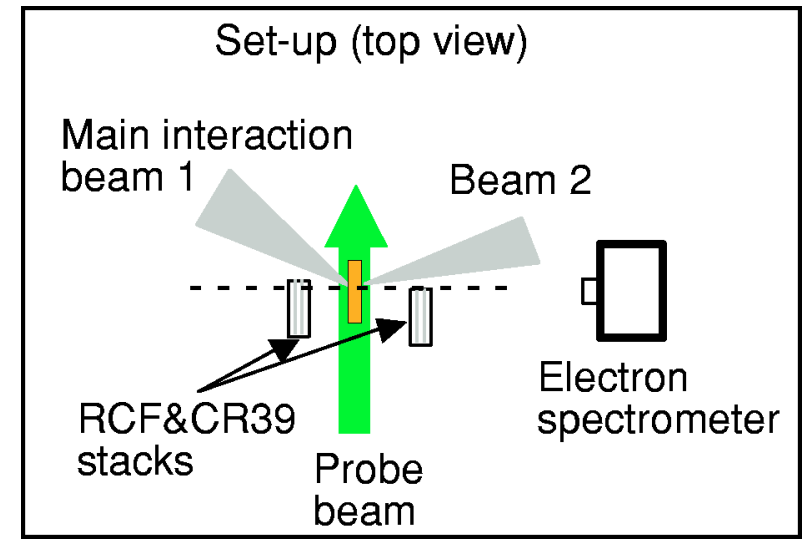

FIG. 1. (Color online) The experimental setup and main diagnostics.

second laser beam. When there is no low-density plasma at the target rear surface, the electron beam is very uniform and no filamentary structure can be observed. The different beam structures can be most easily explained by the Weibel-like instability which can occur in the low-density plasma produced at the rear of the target. Two-dimensional (2D) particle-in-cell (PIC) simulations were also implemented to examine the formation of the magnetic channels in laserplasma interactions using different plasma density scale lengths at the target rear surface. The simulation results agree qualitatively with the experimental observations.

\section{EXPERIMENTAL SETUP}

The experiments were performed at the Rutherford Appleton Laboratory using the Vulcan Nd:glass laser operating in the chirped pulse amplification mode. This laser has a wavelength of $1.054 \mu \mathrm{m}$, a pulse length of $0.9-1.2 \mathrm{psec}$, and a pulse energy between $18 \mathrm{~J}$ and $40 \mathrm{~J}$. In order to generate a plasma on the rear surface of the target, a second beam was used. This was obtained by splitting the laser pulse into two parts: the main interaction beam irradiating the target front surface and a heating beam (beam 2) creating plasma at the target rear surface. The layout is shown in Fig. 1. Both beams were $p$ polarized and focused to $10-\mu \mathrm{m}$-diam spots at $40^{\circ}$ and $20^{\circ}$ to the front and rear surfaces of the foil target by off-axis parabolic mirrors and overlapped to within $10 \mu \mathrm{m}$ using obscuration techniques. The temporal overlap between the laser pulses was measured using a fast-streak camera and was found to be less than $1 \mathrm{psec}$. The targets used were $5 \mathrm{~mm} \times 8 \mathrm{~mm}$ gold foils with a thickness of $20 \mu \mathrm{m}$. A frequency-doubled laser beam $(527 \mathrm{~nm})$ was used to probe the plasma transversely using shadowgraphy.

The main diagnostics used in the experiment were multilayer stacks consisting of radiochromic film (RCF) interleaved with CR39 particle track detectors. The absolutely calibrated RCF detector is sensitive to any ionizing radiation. CR39 nuclear track detectors are only sensitive to energetic ions, so the principal benefit of the multilayer alternating stack is that signals due to electrons and ions can be differentiated unambiguously. A single $12.5-\mu \mathrm{m}$ aluminum foil was used to protect the front piece of RCF from the lowenergy ablated plasma. This layer also functioned as a filter to stop low energy particles (e.g., electrons with energy less than $35 \mathrm{keV}$ will be stopped). The stacks were positioned on both sides of the target, $5 \mathrm{~cm}$ from the target, and aligned so that the faces of the detectors were parallel to the target surface. The main results presented in this paper were obtained from the detector stack at the back side of the target.

The energy spectrum of the electrons was deduced from RCF data. The integrated energy deposited on each layer was obtained by converting the optical density of the exposed film to absorbed dose. The response of each RCF layer as a function of incident electron energy was modeled by a Monte Carlo electron-photon transport code ITS [16]. Then, an unfolding technique based on the YOGI code [17] was used to give the electron energy spectrum.

\section{RESULTS}

Figure 2(a) shows a typical RCF image of the energetic electron beam observed at the back side of the target when there was a plasma generated at rear surface by beam 2 . The intensity of the main beam and heating beam were $10^{19} \mathrm{~W} \mathrm{~cm}^{-2}$ and $5 \times 10^{18} \mathrm{~W} \mathrm{~cm}^{-2}$, respectively. This RCF was at a depth of $1240 \mu \mathrm{m}$ in the stack. No associated ion tracks were found on the CR39 detectors, which indicates that the signals on the RCF are purely due to electrons. The electron beam was emitted at $20^{\circ}$ in the off-normal direction with an $11^{\circ}$ half cone angle. The most interesting characteristic of this beam is that it shows very fine filamentary structures which are $80 \pm 20 \mu \mathrm{m}$ in diameter. All of the other RCF images in the stack show identical structures. The spectrum of the electron beam deduced from RCF images is shown in Fig. 2(b). The total number of electrons in this beam having an energy greater than $100 \mathrm{keV}$ was about $5 \times 10^{12}$ and the total energy in this beam was $\sim 0.4 \mathrm{~J}$ or about $2 \%$ of the incident laser energy.

Off-normal electron beams with similar filamentary structures were also observed when the timing of beam 2 was varied by \pm 2 ps with respect to beam 1 -which implies that the existence of plasmas produced by beam 2 prepulse and/or main pulse at the rear surface with density scale length less than $10 \mu \mathrm{m}[4,13]$ is the important aspect of this interaction. It should be noted that the observed electron beam was not generated by beam 2 since no similar electron beams were ever observed in that direction with beam 1 switched off.

The origin of the observed electron beam must be from the interaction of the main laser beam with the solid target. This is based on observations made from the experiments using only the main laser beam. Without the heating beam, even though there is no long scale length plasma created at the target rear surface, off-normal electron beams were consistently observed on RCF detectors in the back side stack. However, the electron beam in this case is very uniform and no filamentary structure was observable during high-intensity interactions. Figure 3(a) is an image of the electron beam from a typical high intensity shot $\left(I \sim 2 \times 10^{19} \mathrm{~W} \mathrm{~cm}^{-2}\right)$. It should be noted that the electron beam was emitted at the same angle and with the same beam 
a)
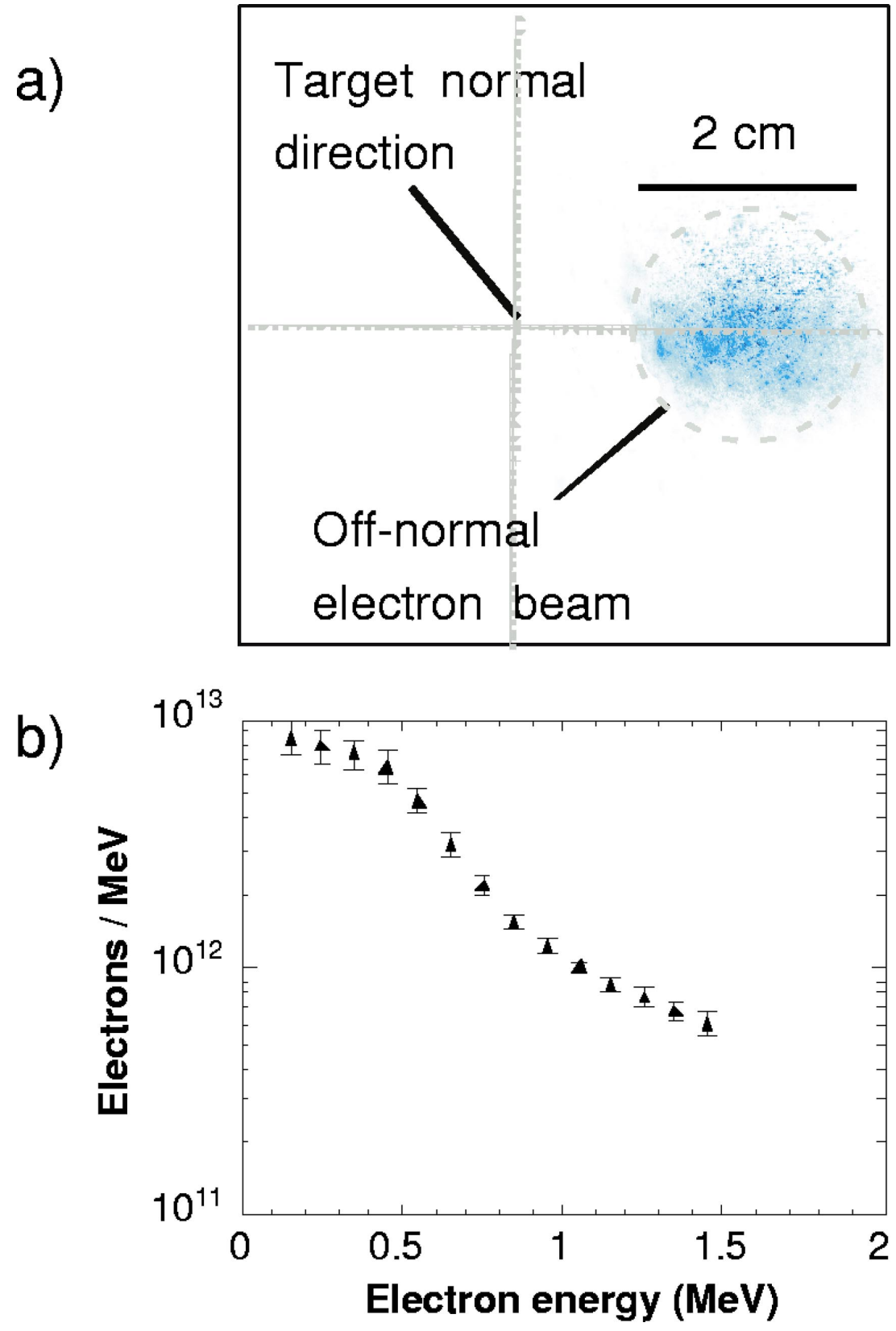

FIG. 2. (Color) Off-normal filamentary electron beam from two laser beam experiments. (a) is a typical electron filamented beam recorded on a RCF layer at the depth of $1240 \mu \mathrm{m}$ in the rear stack; (b) is the energy spectrum of the off-normal electron beam. The intensities of the main interaction beam and beam 2 (heating beam) are $10^{19} \mathrm{~W} \mathrm{~cm}^{-2}$ and $5 \times 10^{18} \mathrm{~W} \mathrm{~cm}^{-2}$, respectively. The two beams were coincident in time.

divergence as that in the two laser case. Electron beam emission at an angle between the laser direction and the target normal has been observed previously and was attributed to effects due to the plasma scale length at the front surface of the target caused by laser prepulse [4].

Only very occasionally (once during this experimental run) could filamentary structure in the electron beam be observed with a single-sided shot on the rear side RCF/CR39 detectors and this was observed to occur at a lower intensity. Figures 3(b) and 3(c) show the RCF images of this particular shot. Figures 3(b) and 3(c) are at different depths in the stack and the laser intensity is about $9 \times 10^{18} \mathrm{~W} \mathrm{~cm}^{-2}$. Signals from two separate regions are clearly visible: an intense beam centered on target normal and an off-normal, less intense beam at $20^{\circ}$ off-normal with angular spread of $11^{\circ}$ half cone angle [as marked in Figs. 3(b) and 3(c)]. From a comparison with CR39 images, the signal in the direction of target normal is primarily due to protons while the offnormal beam is due to electrons. The off-normal electron beam consists of many well-separated electron beamlets such that the beamlet at the center has a size of $\sim 2 \mathrm{~mm}$ (equivalent to a divergence of $0.04 \mathrm{rad}$ ) and is surrounded by smaller beamlets with diameters of the order of $250 \mu \mathrm{m}$ (less than $0.01 \mathrm{rad})$. There are fewer beamlets at greater depths into the stack, indicating that higher-energy components of the electron beam may be less filamented. The total number 

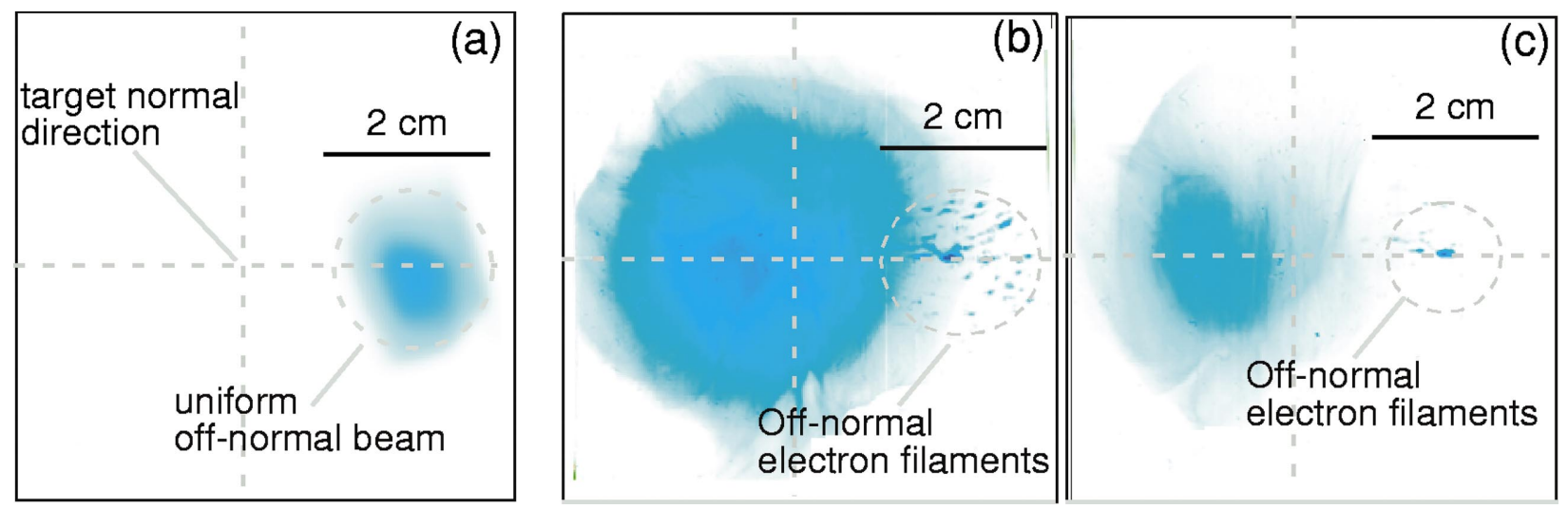

FIG. 3. (Color) Scanned RCF images showing different electron beam structures at different interaction situations with only the main interaction beam. (a) is an RCF image at depths of $2200 \mu \mathrm{m}$ in the stack showing a uniform off-normal electron beam at the laser intensity of $2 \times 10^{19} \mathrm{~W} \mathrm{~cm}^{-2}$. (b) and (c) at depths of $680 \mu \mathrm{m}$ and $1240 \mu \mathrm{m}$ inside the stack, show the large electron filaments at $\sim 20^{\circ}$ off-normal direction (marked by dotted circle) at a laser intensity $\sim 9 \times 10^{18} \mathrm{~W} \mathrm{~cm}^{-2}$.

of electrons with energy greater than $100 \mathrm{keV}$ is significantly less than that from the two-beam case. This difference is likely due to the lack of low-density, long-scale-length plasma at the rear surface of the target which may enhance the amount of target charging and reduce the number of electrons which can escape from the target. Once again, the similarity of the electrons beams shown in Figs. 2 and 3 (i.e., having the same emission angle and similar beam divergence) of these off-normal electron beams is clear evidence that the off-normal electron beams observed with heating laser beam originate from the interactions of the main laser beam.

Note that in our experiments we are only measuring the minority of energetic electrons which are able to escape from the plasma formed during the interaction. As the target charges up because of the escape of these electrons the emission of the remainder of the hot electron population will be affected (especially in the cases without an extended plasma at the rear surface) [18].

\section{ELECTRON BEAM FILAMENTATION DUE TO THE WEIBEL-LIKE INSTABILITY}

The finer-scale filamentation in the electron beams always observed in the two-beam experiments is clearly caused by the long plasma density scale length at the rear surface of the target. As the plasma density at the back of the foil varies from solid density $\left(10^{23} \mathrm{~cm}^{-3}\right)$ to vacuum $\left(10^{13} \mathrm{~cm}^{-3}\right)$, there exists a significant region over which the beam density $\left(<10^{21} \mathrm{~cm}^{-3}\right)$ is comparable to the background density. Simple theory suggests that the growth rate of the Weibellike instability increases dramatically when the background density is similar to the electron beam density. Simultaneous optical transition radiation (OTR) measurements using gated optical imaging showed that the diameter of the intense selfemission signal due to the electron beam exiting the target rear surface in single-beam experiments is about $40-50 \mu \mathrm{m}$. Consequently, given the number of the electron filaments recorded on the RCF images, it is estimated that the wavelength associated with the instability is approximately
$0.75 \pm 0.25 \mu \mathrm{m}$ in our experiments. It should be noted that this is approximately the collisionless skin depth at an electron density in the range of $10^{20} \mathrm{~cm}^{-3}$, which is the estimated density of the electron beam.

To examine this further we performed 2D PIC simulations using the OSIRIS code [19], with uniform laser irradiation of a uniform overdense plasma with density of 2.2 $\times 10^{22} \mathrm{~cm}^{-3}$ having a sharp boundary at the front surface. Two different density profiles at the target rear surface were assumed: i.e., a sharp boundary and a longer plasma scale length. The laser was linearly polarized and was incident along the normal direction, rising to a constant intensity of $10^{19} \mathrm{~W} \mathrm{~cm}^{-2}$ in $6 \mathrm{fsec}$. The system size was $9 \mu \mathrm{m}$ square and the plasma length was $5 \mu \mathrm{m}$. Our simulation results suggest that shallow discrete magnetic channels not only occur at the critical density surface at the front surface, but also appear with much larger spatial scale at the rear surface when the beam density is comparable to the background plasma density. Figure 4 shows the quasistatic magnetic fields at a time of $144 \mathrm{fs}$ for two different density profiles at the rear surface. Figure 4(a) is the result of a simulation using a target with a very sharp rear boundary, and Fig. 4(b) is the result of a simulation which uses a much longer density scale length such that the plasma density drops linearly from $20 n_{c}$ to $n_{c}$ in a distance of $14 c / \omega_{0}$ along the direction of laser propagation. With a long plasma scale length at the rear surface, the Weibel instability at the rear region is much more pronounced, showing maximum growth at scale sizes of 3-5 times larger than that at the front surface. This is in reasonable agreement with our experimental observations.

As mentioned previously, filamentation of electron beams due to Weibel-like instabilities has been studied numerically using both PIC and Fokker-Planck hybrid codes [9-11]. Linear analysis of the Weibel instability based on relativistic two-stream or relativistic Vlasov models $[9,11,14]$ suggests that plasma kinetic effects (collisional dissipation) play an important role in its evolution. It was found that the growth rate of large wavelength modes in the collisional case is higher than that in the collisionless case. 2D PIC simulations [9] have also shown that the spectral peak of the growth rate shifts to longer-wavelength modes in the more collisional 


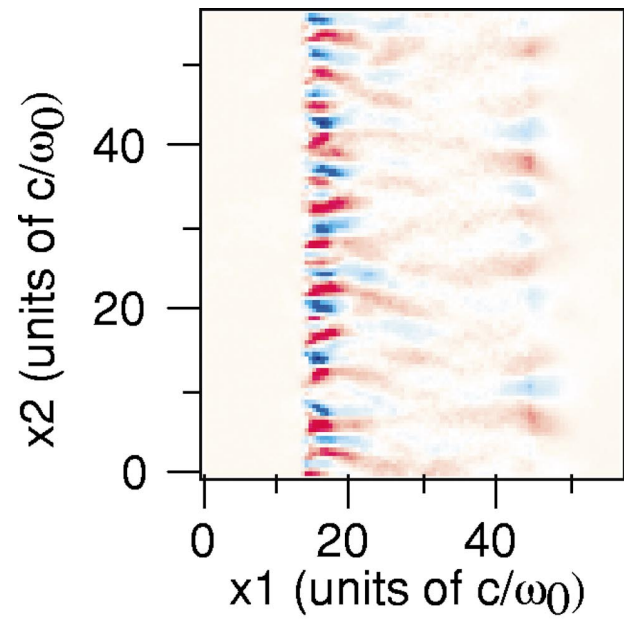

(a)

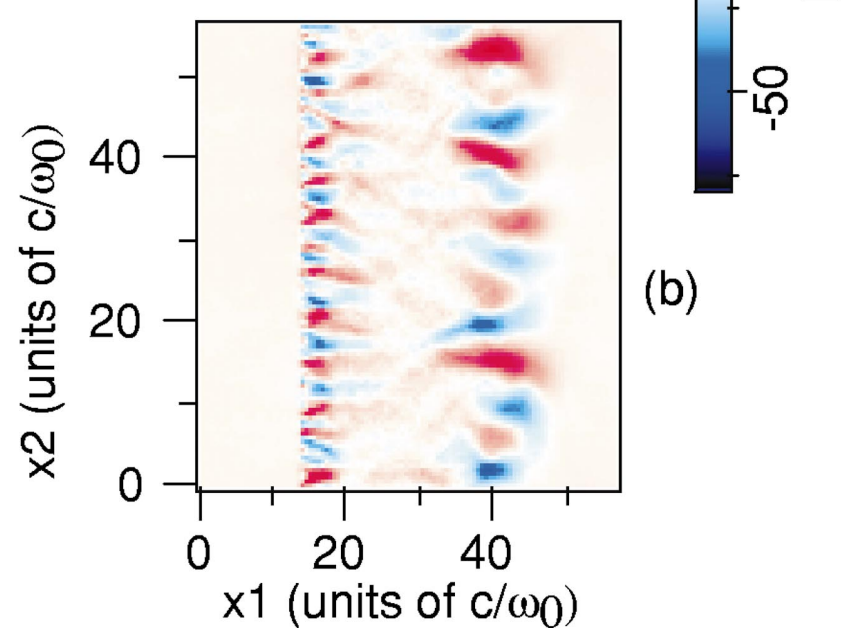

FIG. 4. (Color) Simulation results of the structure of the time averaged quasistatic magnetic fields at $144 \mathrm{fs}$ with different density scale lengths at the target rear surface. (a) is with a very sharp drop from $20 n_{c}$ to 0 within $0.2 c / \omega_{0}$ and (b) is with a slow ramp from $20 n_{c}$ to $n_{c}$ within $14 c / \omega_{0}$. Here $x_{1}$ is the coordinate along laser direction of propagation. A linearly polarized laser pulse with an intensity $\sim 10^{19} \mathrm{~W} / \mathrm{cm}^{2}$ is incident from the left boundary. The plasma and laser light are uniform in the $x_{2}$ direction. One unit in space corresponds to $0.16 \mu \mathrm{m}$.

case. In our experiments, the background plasma can be assumed to be cold and highly collisional-note that if $\sim 10 \%$ of the laser energy is deposited in the interior, the internal plasma temperature is likely to be less than $100 \mathrm{eV}$. Consequently, plasma kinetic effects may be important in the evolution of this instability, which may result in the rapid growth of longer wavelength modes. The large electron beamlets observed occasionally in the single-beam experiments at lower intensity may be as a result of the growth of longerwavelength modes in a colder, more collisional plasma.

Recently, Silva et al. [20] showed that the growth rate of the Weibel instability is dependent on the ratio of the beam density to background plasma density, the perpendicular temperature of the beam, and the beam energy. The threshold for the Weibel instability to occur for a cold relativistic beam in a cold plasma $\left(p_{\perp} / m_{e} c \ll 1, p_{\|} / m_{e} c \gg 1\right)$ was derived from relativistic kinetic theory to be

$$
\frac{n_{b}}{n_{e}}>\gamma_{b}\left(\frac{p_{\perp}}{p_{\|}}\right)^{2},
$$

where $n_{b}$ is the beam density, $n_{e}$ is the background plasma density, $\gamma_{b}$ is the relativistic factor of the electron beam, and $p_{\|}$and $p_{\perp}$ are the electron beam momenta along the beam direction and transverse to the beam, respectively. This indicates that the threshold for the Weibel instability is increased at higher laser intensities due to the larger value of $\gamma_{b}$ as well as a possible increase in the transverse temperature of the beam. In addition, the growth rate may be decreased because of reduced collisionality if the target is heated to higher temperatures. The combination of these effects may explain why this instability was almost never observed in our experiments using single beam at high intensities. Indeed, the "coarse" electron filaments without target rear surface heating were only observed once in the experiments, while off-normal "uniform beams" were usually observed. Further experiments are required to determine conclusively whether the observed filaments in the single-beam case were due to laser intensity, prepulse levels, or target conditions.

Besides the well-known Weibel-like instability, there are other mechanisms which may also produce filamented electron beams at the laser-plasma interface. For example, the surface instability [21], which is associated with deformations at the laser-plasma interface at high intensity, can potentially generate fast electron filaments. It has been suggested that a "smooth" density deformation as wide as the laser spot, such as that produced during hole boring process, leads to high absorption and to collimation of fast electrons due to a geometrical "funnel" effect. Due to the appearance of small-scale deformations at high intensity, several electron filaments, each of them correlated with a small-scale deformation, may appear. It is possible that the surface instability may cause the large-scale structure observed in the singlebeam case. However, considering the consistent observation of uniform off-normal electron beams using only main interaction laser beam, it is quite likely that this instability mechanism does not play a significant role in the observed electron filaments in our experiments. Indeed, the fine-scale structure observed in the two-beam interactions is not due to a change in the laser-plasma interaction but rather a change in the parameters of the interaction of the electron beam with the plasma.

\section{CONCLUSIONS}

Electron beam production and propagation have been studied with 1-psec laser pulses interacting with thin gold targets at intensities greater than $5 \times 10^{18} \mathrm{~W} / \mathrm{cm}^{2}$. Filamentary emission of relativistic electron beams leaving the rear surface of the target has been experimentally observed. This is the first such observation. It was shown that the most important condition in which electron beam filamentation due to Weibel-like instabilities becomes a factor are situations where large regions of low-density plasma exist (i.e., with the plasma density comparable to the beam density). Electron beam propagation in cold collisional plasmas may also enhance this instability. These observations are in agree- 
ment with theory and computer simulation results. Our experimental results also suggest that Weibel-like instabilities may not be important for the fast-ignitor scheme if experiments are designed so that the background plasma density is greater than the beam density, which results in a significant increase of the instability threshold and a decrease in the growth rate. Such effects are also less important at higher intensities because of larger transverse temperatures in the generated electron beam.

Such techniques should also be useful for further systematic studies of electron beam production and propagation in high-intensity laser-plasma interaction experiments.

\section{ACKNOWLEDGMENTS}

The authors acknowledge the assistance of the staff of the Central Laser Facility of the Rutherford Appleton Laboratory in the execution of this work as well as the support of the UK Engineering and Physical Sciences Research Council (EPSRC). We gratefully acknowledge the OSIRIS consortium which consists of UCLA/IST(Portugal)/USC for the use of OSIRIS. M.S.W. is partially supported by the Overseas Research Students Awards Scheme, Universities UK.
[1] M. Tabak et al., Phys. Plasmas 1, 1626 (1994).

[2] R. Kodama et al., Nature (London) 412, 798 (2001); R. Kodama et al., ibid. 418, 933 (2002).

[3] M. H. Key et al., Phys. Plasmas 5, 1966 (1998); P. A. Norreys et al., ibid. 6, 2150 (1999); T. Hall et al., Phys. Rev. Lett. 81, 1003 (1998).

[4] M. I. K. Santala et al., Phys. Rev. Lett. 84, 1459 (2000).

[5] S. C. Wilks and W. L. Kruer, IEEE J. Quantum Electron. 33, 1954 (1997); F. Brunel, Phys. Rev. Lett. 59, 52 (1987).

[6] S. C. Wilks et al., Phys. Rev. Lett. 69, 1383 (1992).

[7] E. S. Weibel, Phys. Rev. Lett. 2, 83 (1959).

[8] F. Califano et al., Phys. Rev. E 56, 963 (1997); F. Califano et al., ibid. 57, 7048 (1998).

[9] Y. Sentoku et al., Phys. Plasmas 7, 689 (2000).

[10] Y. Sentoku et al., Phys. Rev. E 65, 046408 (2002); Y. Sentoku et al., Phys. Rev. Lett. 90, 155001 (2003); M. Honda, J. Meyer-ter-Vehn, and A. Pukhov, Phys. Plasmas 7, 1302 (2000); B. F. Lasinski et al., ibid. 6, 2041 (1999).
[11] L. Gremillet et al., Phys. Plasmas 9, 941 (2002); J. Davies et al., Phys. Rev. E 56, 7193 (1997).

[12] M. Borghesi et al., Phys. Rev. Lett. 83, 4309 (1999); L. Gremillet et al., ibid. 83, 5015 (1999); M. Tatarakis et al., ibid. 90, 175001 (2003).

[13] M. Tatarakis et al., Phys. Rev. Lett. 81, 999 (1998).

[14] E. L. Clark et al., Phys. Rev. Lett. 84, 670 (2000); M. Zepf et al., ibid. 90, 064801 (2003).

[15] J. Fuchs et al., Phys. Rev. Lett. 91, 255002 (2003).

[16] J. A. Halbleib, Nucl. Sci. Eng. 92, 338 (1986).

[17] G. A. Calson et al., Nucl. Instrum. Methods Phys. Res. B 62, 269 (1991); S. G. Gorbics and N. R. Pereira, Rev. Sci. Instrum. 64, 1835 (1993).

[18] F. N. Beg et al., Phys. Rev. Lett. 92, 095001 (2004).

[19] R. Hemker, Ph.D. dissertation, UCLA, 2000.

[20] L. O. Silva et al., Phys. Plasmas 9, 2458 (2002).

[21] A. Macchi et al., Nucl. Fusion 43, 362 (2003). 